\title{
ON A CANONICAL CONFORMAL MAPPING OF J. L. WALSH
}

BY

\author{
JAMES A. JENKINS
}

1. In a recent paper [3] Walsh has given a type of canonical conformal mapping for plane domains of finite connectivity which may be regarded as the culmination of results of de la Vallée Poussin and Julia on canonical conformal mappings associated with level curves of polynomials and rational functions. On the one hand Walsh allows a class of transcendental functions replacing the rational functions of the earlier works. On the other the striking features of his paper are, first, the completeness of the representation obtained as compared with the results of de la Vallee Poussin and Julia and, second, the fact that with an appropriate interpretation the canonical mapping for a given domain is unique. It is of considerable interest that Walsh's results can be obtained by use of the method of quadratic differentials, indeed by a suitable development of considerations given in a paper [2] by the author and D. C. Spencer. The exposition of these ideas is the purpose of the present paper.

2. TheOREM 1. Let $D$ be a domain on the z-sphere with boundary components $B_{1}, B_{2}, \cdots, B_{\mu} ; C_{1}, C_{2}, \cdots, C_{\nu}, \mu \nu \neq 0$. Then there exists a conformal mapping of $D$ onto a domain $\Delta$ on the $Z$-sphere where $\Delta$ is defined by

$$
\begin{aligned}
1<|T(Z)|<e^{1 / \tau}, \quad T(Z) & \equiv \frac{A\left(Z-a_{1}\right)^{M_{1}}\left(Z-a_{2}\right)^{M_{2}} \cdots\left(Z-a_{\mu}\right)^{M_{\mu}}}{\left(Z-b_{1}\right)^{N_{1}}\left(Z-b_{2}\right)^{N_{2}} \cdots\left(Z-b_{\nu}\right)^{N_{\nu}}}, \\
\sum_{j=1}^{\mu} M_{j} & =\sum_{j=1}^{\nu} N_{j}=1, \quad \tau>0 .
\end{aligned}
$$

The exponents $M_{j}$ and $N_{j}$ are positive but need not be rational. The locus $|T(Z)|$ $=1$ consists of $\mu$ mutually disjoint Jordan curves, respective images of the $B_{j}$, which separate $\Delta$ from the $a_{j}$; the locus $|T(Z)|=e^{1 / r}$ consists of $\nu$ mutually disjoint Jordan curves, respective images of the $C_{j}$, which separate $\Delta$ from the $b_{j}$.

In his corresponding statement [3, p. 129] Walsh includes the remark that if the $B_{j}, C_{j}$ are Jordan curves the mapping can be extended to a homeomorphism between $\bar{D}$ and $\bar{\Delta}$. As appears in his proof this follows at once from standard results on boundary correspondence when Theorem 1 is established.

In $[2, \S 8]$ is treated a canonical conformal mapping of a multiply-connected domain obtained from a regular positive quadratic differential given on the domain. (In that paper negative quadratic differentials were used but

Received by the editors November 10, 1956. 
this is inessential.) If one uses a quadratic differential derived from the harmonic measure of the set of curves $C_{j}$ with respect to the domain $D$ it will be found that the mapping obtained is that of Theorem 1. Here we will develop the details from the beginning for the sake of completeness. Actually in the proof of Theorem 1 one need introduce only linear differentials but in a more general context quadratic differentials would intervene.

Let $\omega(z)$ be the harmonic measure of $\bigcup_{j=1}^{\nu} C_{j}$ with respect to $D$ and let $\varpi(z)$ be a conjugate of $\omega(z)$. Then $f(z)=\omega(z)+i \varpi(z)$ will be analytic but not single-valued in $D$ while $f^{\prime}(z)$ will be single-valued. Let us join the boundary components $B_{1}, B_{2}, \cdots, B_{\mu}$ to a point $P$ in $D$ by a finite number of analytic arcs such that just one arc ends on each boundary component and the boundary components $C_{1}, C_{2}, \cdots, C_{\nu}$ to a point $P^{\prime}$ in $D$ by a finite number of analytic arcs such that just one arc ends on each boundary component in such a way that on deleting these arcs from $D$ we obtain a single doublyconnected domain $D^{\prime}$. Finally we join the point $P$ to the point $P^{\prime}$ by an analytic arc in $D^{\prime}$ so that on deleting it from $D^{\prime}$ we obtain a single simply-connected domain $D^{*}$. This is mapped by the function if $(z)$ (which is singlevalued on $D^{*}$ ) onto a portion of Riemann surface $\Re^{\prime}$ lying, say, over the $w$ plane. If we identify boundary points of $\Re^{\prime}$ arising from the same point of $D$ we obtain a conformal model of $D$. Under the preceding mapping a boundary component $B_{j}$ goes into a segment over the real $w$-axis of length say $\sigma_{j}^{\prime}$. A boundary component $C_{j}$ similarly goes into a segment lying above the line $\Im w=1$ of length say $\tau_{j}^{\prime}$. By a well known property of the harmonic measure

$$
\sum_{j=1}^{\mu} \sigma_{j}^{\prime}=\sum_{j=1}^{\nu} \tau_{j}^{\prime}=2 \pi \tau
$$

where $\tau$ is a certain positive number.

Consider now the function $i(2 \pi \tau)^{-1} f(z)$. As before it maps $D^{*}$ onto a portion of Riemann surface $\Re$ lying, say, over the $\zeta$-plane. Let $B_{j}$ go into the segment $\Sigma_{j}$

$$
s_{j} \leqq \zeta \leqq s_{j}+\sigma_{j}
$$

where $\sigma_{j}=(2 \pi \tau)^{-1} \sigma_{j}^{\prime}$ and the end points represent the same point on $B_{j}$. Let $C_{j}$ go into the segment $\mathrm{T}_{j}$

$$
\Im \zeta=(2 \pi \tau)^{-1}, \quad t_{j} \leqq \Re \zeta \leqq t_{j}+\tau_{j}
$$

where $\tau_{j}=(2 \pi \tau)^{-1} \tau_{j}^{\prime}$ and the end points represent the same point on $C_{j}$.

Now we adjoin to $\Re$ along the segment $\Sigma_{j}, j=1, \cdots, \mu$, the half strip $F_{j}$

$$
\Im \zeta<0, \quad s_{j} \leqq \Re \zeta \leqq s_{j}+\sigma_{j} .
$$

Also we adjoin to $\Re$ along the segment $\mathrm{T}_{j}, j=1, \cdots, \nu$, the half strip $G_{j}$

$$
\Im \zeta>(2 \pi \tau)^{-1}, \quad t_{j} \leqq \Re_{i} \zeta \leqq t_{j}+\tau_{j} .
$$


From the covering surface so obtained we produce an abstract Riemann surface $\subseteq$ by

(i) performing the identifications arising from the mapping $i(2 \pi \tau)^{-1} f(z)$,

(ii) identifying each two boundary points of $F_{j}$ for which $\Im \zeta$ has a given value, assigning there as local uniformizing parameter the parameter obtained by covering of the $\zeta$-plane, $j=1, \cdots, \mu$,

(iii) identifying each two boundary points of $G_{j}$ for which $\Im \zeta$ has a given value, assigning there as local uniformizing parameter the parameter obtained by covering of the $\zeta$-plane, $j=1, \cdots, \nu$,

(iv) assigning a point $P_{j}$ corresponding to the point at infinity on each of $F_{j}, j=1, \cdots, \mu$, at which we assign the local uniformizing parameter $\exp \left(-2 \pi i \sigma_{j}^{-1} \zeta\right)$,

(v) assigning a point $Q_{j}$ corresponding to the point at infinity on each of $G_{j}, j=1, \cdots, \nu$, at which we assign the local uniformizing parameter $\exp \left(2 \pi i \tau_{j}^{-1} \zeta\right)$.

The surface $\mathfrak{S}$ is seen at once to be simply-connected and of elliptic type. Let it be mapped conformally on the $Z$-sphere. By this mapping to the points $P_{j}, j=1, \cdots, \mu$, correspond points $a_{j}$ and to the points $Q_{j}$, $j=1, \cdots, \nu$, correspond points $b_{j}$. We may assume all the $a_{j}, b_{j}$ finite (although corresponding considerations would apply if one of them were the point at infinity). As a function of $Z, \zeta$ is of course not single-valued but $d \zeta$ is. It is thus a linear differential on the $Z$-sphere and is regular except at the points $a_{j}, j=1, \cdots, \mu$ and $b_{j}, j=1, \cdots, \nu$. At $a_{j}$ we have the expansion

$$
d \zeta=\left(\frac{i \sigma_{j}}{2 \pi} \frac{1}{Z-a_{j}}+\text { regular terms }\right) d Z
$$

and at $b_{j}$ the expansion

$$
d \zeta=\left(\frac{-i \tau_{j}}{2 \pi} \frac{1}{Z-b_{j}}+\text { regular terms }\right) d Z .
$$

Thus

$$
d \zeta=\frac{i}{2 \pi}\left\{\sum_{j=1}^{\mu} \frac{\sigma_{j}}{Z-a_{j}}-\sum_{j=1}^{\nu} \frac{\tau_{j}}{Z-b_{j}}\right\} d Z .
$$

(Note that $d \zeta$ is regular at $Z=\infty$.) Moreover

$$
\zeta=\frac{i}{2 \pi}\left\{\sum_{j=1}^{\mu} \sigma_{j} \log \left(Z-a_{j}\right)-\sum_{j=1}^{\nu} \tau_{j} \log \left(Z-b_{j}\right)\right\}+K
$$

where $K$ is constant. Take now

$$
T(Z)=\exp (-2 \pi i \zeta)
$$

and set $\sigma_{j}=M_{j}, \tau_{j}=N_{j}, \exp (-2 \pi i K)=A$. Then 


$$
T(Z) \equiv \frac{A\left(Z-a_{1}\right)^{M_{1}}\left(Z-a_{2}\right)^{M_{2}} \cdots\left(Z-a_{\mu}\right)^{M_{\mu}}}{\left(Z-b_{1}\right)^{N_{1}}\left(Z-b_{2}\right)^{N_{2}} \cdots\left(Z-b_{\nu}\right)^{N_{\nu}}}
$$

with $\sum_{j=1}^{\mu} M_{j}=\sum_{j=1}^{\nu} N_{j}=1$. On the other hand, on the curves $B_{j}$, $j=1, \cdots, \mu$, we have $\Im \zeta=0$; on the curves $C_{j}, j=1, \cdots, \nu$, we have $\Im \zeta$ $=i(2 \pi \tau)^{-1}$. Thus on the images of the first set of curves $|T(Z)|=1$ and on the images of the second set of curves $|T(Z)|=\exp (1 / \tau)$. It is seen at once that these are the only points at which these equalities hold and that the points of $D$ go into the points for which

$$
1<|T(Z)|<\exp (1 / \tau) \text {. }
$$

This completes the proof of Theorem 1 .

3 . We give now a proof of the uniqueness result corresponding to Theorem $1[3$, p. 137].

Theorem 2. Let D be a domain on the z-sphere defined by the inequalities

$$
\begin{gathered}
1<|R(z)|<e^{1 / \tau}, \quad R(z) \equiv \frac{A\left(z-a_{1}\right)^{m_{1}} \cdots\left(z-a_{\mu}\right)^{m_{\mu}}}{\left(z-b_{1}\right)^{n_{1}} \cdots\left(z-b_{\nu}\right)^{n_{\nu}}}, \\
\sum_{j=1}^{\mu} m_{j}=\sum_{=1}^{\nu} n_{j}=1,
\end{gathered}
$$

and whose boundary consists of mutually disjoint Jordan curves $B_{1}, B_{2}, \cdots, B_{\mu}$, $C_{1}^{\prime}, C_{2}, \cdots, C_{\nu}$, where $B_{j}$ separates $a_{j}$ from $D$ and $C_{j}$ separates $b_{j}$ from $D$. Let $\Delta$ be a domain on the $Z$-sphere defined by the inequalities

$$
\begin{gathered}
1<\left|R_{1}(Z)\right|<e^{1 / \tau^{\prime}}, \quad R_{1}(Z) \equiv \frac{A^{\prime}\left(Z-a_{1}^{\prime}\right)^{m_{1}{ }^{\prime}} \cdots\left(Z-a_{\mu}^{\prime}\right)^{m_{\mu^{\prime}}}}{\left(Z-b_{1}^{\prime}\right)^{n_{1}} \cdots\left(Z-b_{\nu}^{\prime}\right)^{n_{\nu^{\prime}}}}, \\
\sum_{j=1}^{\mu} m_{j}^{\prime}=\sum_{j=1}^{\nu} n_{j}^{\prime}=1
\end{gathered}
$$

and whose boundary consists of mutually disjoint Jordan curves $B_{1}^{\prime}, B_{2}^{\prime}, \cdots, B_{\mu}^{\prime}$, $C_{1}^{\prime}, C_{2}^{\prime}, \cdots, C_{\nu}^{\prime}$, where $B_{j}^{\prime}$ separates $a_{j}^{\prime}$ from $\Delta$ and $C_{j}^{\prime}$ separates $b_{j}^{\prime}$ from $\Delta$. If there exists $a(1,1)$ conformal mapping of $D$ onto $\Delta$ so that each $B_{j}$ corresponds to $B_{j}^{\prime}, j=1, \cdots, \mu$, and each $C_{j}$ corresponds to $C_{j}^{\prime}, j=1, \cdots, \nu$, then the mapping is a linear transformation of the complex variable $z$, defined throughout the z-and $Z$-spheres. We have $\tau=\tau^{\prime}, m_{j}=m_{j}^{\prime}, n_{j}=n_{j}^{\prime}$. If $\arg A^{\prime}$ is suitably chosen we have $R(z) \equiv R_{1}(Z)$.

Let $Z=g(z)$ be the conformal mapping of Theorem 2. Define

$$
\begin{gathered}
u(z)=\sum_{j=1}^{\mu} m_{j} \log \left|z-a_{j}\right|-\sum_{j=1}^{\nu} n_{j} \log \left|z-b_{j}\right|+\log |A|, \\
U(Z)=\sum_{j=1}^{\mu} m_{j}^{\prime} \log \left|Z-a_{j}^{\prime}\right|-\sum_{j=1}^{\nu} n_{j}^{\prime} \log \left|Z-b_{j}^{\prime}\right|+\log \left|A^{\prime}\right| .
\end{gathered}
$$


The function $u(z)$ is single-valued and harmonic in $D$, takes the value 0 on the curves $B_{j}, j=1, \cdots, \mu$ and the value $1 / \tau$ on the curves $C_{j}, j=1, \cdots, \nu$. It is thus equal to $1 / \tau$ times the harmonic measure of the set of curves $C_{j}$, $j=1, \cdots, \nu$ with respect to $D$. Similarly $U(Z)$ is $1 / \tau^{\prime}$ times the harmonic measure of the set of curves $C_{j}^{\prime}, j=1, \cdots, \nu$, with respect to $\Delta$. Thus $\tau u(z)$ $=\tau^{\prime} U(g(z))$. Now $2 \pi m_{j}$ is the period of a conjugate of $u(z)$ about $B_{j},-2 \pi n_{j}$ the period of a conjugate of $u(z)$ about $C_{j}$ while $2 \pi m_{j}^{\prime},-2 \pi n_{j}^{\prime}$ are the corresponding periods for $U(Z)$. Thus

$$
\begin{aligned}
\tau m_{j} & =\tau^{\prime} m_{j}^{\prime}, \\
\tau n_{j} & =\tau^{\prime} n_{j}^{\prime},
\end{aligned}
$$$$
\begin{aligned}
& j=1, \cdots, \mu \\
& j=1, \cdots, \nu .
\end{aligned}
$$

Adding the first set of equations we obtain

$$
\tau=\tau^{\prime} \text {. }
$$

This means

$$
\begin{aligned}
m_{j} & =m_{j}^{\prime}, \\
n_{j} & =n_{j}^{\prime},
\end{aligned}
$$$$
\begin{aligned}
j & =1, \cdots, \mu \\
i & =1, \cdots, \nu
\end{aligned}
$$

while

$$
u(z)=U(g(z))
$$

Setting now

$$
\begin{aligned}
d \zeta & =\left(\sum_{j=1}^{\mu} \frac{m_{j}}{z-a_{j}}-\sum_{j=1}^{\nu} \frac{n_{j}}{z-b_{j}}\right) d z, \\
d \zeta^{\prime} & =\left(\sum_{j=1}^{\mu} \frac{m_{j}}{Z-a_{j}^{\prime}}-\sum_{j=1}^{\nu} \frac{n_{j}}{Z-b_{j}^{\prime}}\right) d Z
\end{aligned}
$$

we have at once that $d \zeta$ and $d \zeta^{\prime}$ are corresponding differentials under the mapping $g$. Defining on $D$ and $\Delta$ the respective metrics $|d \zeta|$ and $\left|d \zeta^{\prime}\right|$ the mapping $g$ becomes isometric.

Let now $\Gamma$ and $\Gamma^{\prime}$ be a pair of corresponding boundary components of $D$ and $\Delta$; let $c$ and $c^{\prime}$ be the distinguished points they separate from $D$ and $\Delta$. Evidently $\Gamma$ and $\Gamma^{\prime}$ are closed trajectories respectively of the quadratic differentials $-d \zeta^{2}$ and $-\left(d \zeta^{\prime}\right)^{2}$. Let $O$ and $\Omega$ be the domains bounded respectively by $\Gamma$ and $\Gamma^{\prime}$ in the complements of $D$ and $\Delta$. Since $O(\Omega)$ contains no pole of $-d \zeta^{2}\left(-\left(d \zeta^{\prime}\right)^{2}\right)$ other than $c\left(c^{\prime}\right)$, by the Basic Structure Theorem [1] $O(\Omega)$ is a subdomain of a circle domain associated with $-d \zeta^{2}\left(-\left(d \zeta^{\prime}\right)^{2}\right)$. Consider $z$ on $\Gamma$ and the corresponding point $g(z)$ on $\Gamma^{\prime}$. The portion of orthogonal trajectory of $-d \zeta^{2}\left(-\left(d \zeta^{\prime}\right)^{2}\right)$ entering $\bar{O}(\bar{\Omega})$ at $z(g(z))$ is a half-open arc $\Lambda_{z}\left(\Lambda_{z}^{\prime}\right)$ with limiting end point at $c\left(c^{\prime}\right)$. We extend the mapping $g$ to the set $O$ by mapping the point of $\Lambda_{z}$ at a given distance from $z$ in the metric $|d \zeta|$ onto the point of $\Lambda_{z}^{\prime}$ at the same distance from $g(z)$ in the metric $\left|d \zeta^{\prime}\right|$ and map $c$ 
onto $c^{\prime}$. This clearly gives a $(1,1)$ mapping of $O$ onto $\Omega$. At all points of $\bar{O}$ but $c$ the mapping is isometric for the metrics $|d \zeta|,\left|d \zeta^{\prime}\right|$ thus conformal. Finally by the Riemann theorem on removable singularities the mapping is conformal at $c$. Carrying out this construction for all corresponding pairs of domains complementary to $D$ and $\Delta$ we extend $g$ to a conformal mapping of the $z$-sphere onto the $Z$-sphere which is thus a linear transformation.

Finally by Equation (2) we have

$$
|R(z)|=\left|R_{1}(g(z))\right| \text {. }
$$

Thus for a suitable choice of $\arg A^{\prime}$ we have

$$
R(z) \equiv R_{1}(g(z)) \text {. }
$$

This completes the proof of Theorem 2.

3. Walsh gave also an analogue to Theorem 1 when the boundary components $B_{j}, j=1, \cdots, \mu$, are allowed to shrink to points [3, p. 140].

THEOREM 3. Let $D$ be a domain on the z-sphere with boundary components $C_{1}, C_{2}, \cdots, C_{\nu}$, let $\alpha_{1}, \alpha_{2}, \cdots, \alpha_{\mu}$ be arbitrary distinct points of $D$ and let $M_{1}, M_{2}, \cdots, M_{\mu}$ be arbitrary positive numbers which satisfy $\sum_{j=1}^{\mu} M_{j}=1$. Then there exists a conformal mapping of $D$ onto a domain $\Delta$ on the $Z$-sphere where $\Delta$ is defined by

$$
\begin{aligned}
|T(Z)|<1, \quad T(Z) & \equiv \frac{A\left(Z-a_{1}\right)^{M_{1}}\left(Z-a_{2}\right)^{M_{2}} \cdots\left(Z-a_{\mu}\right)^{M_{\mu}}}{\left(Z-b_{1}\right)^{N_{1}}\left(Z-b_{2}\right)^{N_{2}} \cdots\left(Z-b_{\nu}\right)^{N_{\nu}}}, \\
N_{j} & >0, \quad \sum_{j=1}^{\nu} N_{j}=1 .
\end{aligned}
$$

The $a_{j}$ are the respective images of the $\alpha_{j}$, the locus $|T(Z)|=1$ consists of $\nu$ mutually disjoint analytic Jordan curves, which are respective images of the $C_{j}$ and which separate $\Delta$ from the $b_{j}$.

A remark on boundary correspondence holds as for Theorem 1 .

Theorem 3 is proved just as Theorem 1 except the harmonic measure is replaced by the function

$$
M_{1} g_{1}(z)+M_{2} g_{2}(z)+\cdots+M_{\mu} g_{\mu}(z)
$$

where $g_{j}(z)$ is the Green's function of $D$ with pole at $\alpha_{j}, j=1, \cdots, \mu$.

4. The uniqueness result corresponding to Theorem 3 is $[3$, p. 143]

THEOREM 4. Let $D$ be a domain on the z-sphere defined by the inequality

$$
\begin{gathered}
|R(z)|<1, \quad R(z) \equiv \frac{A\left(z-a_{1}\right)^{m_{1}}\left(z-a_{2}\right)^{m_{2}} \cdots\left(z-a_{\mu}\right)^{m_{\mu}}}{\left(z-b_{1}\right)^{n_{1}}\left(z-b_{2}\right)^{n_{2}} \cdots\left(z-b_{\nu}\right)^{n_{\nu}}}, \\
m_{j}>0, \quad \sum_{j=1}^{\mu} m_{j}=\sum_{j=1}^{\nu} n_{j}=1,
\end{gathered}
$$


and whose boundary consists of mutually disjoint Jordan curves $C_{1}, C_{2}, \cdots, C_{\nu}$ which separate the $b_{j}$ respectively from $D$. Let $\Delta$ be a domain on the $Z$-sphere defined by the inequality

$$
\begin{aligned}
\left|R_{1}(Z)\right|<1, \quad R_{1}(Z) \equiv & \frac{A^{\prime}\left(Z-a_{1}^{\prime}\right)^{m_{1}}\left(Z-a_{2}^{\prime}\right)^{m_{2}} \cdots\left(Z-a_{\mu}^{\prime}\right)^{m_{\mu}}}{\left(Z-b_{1}^{\prime}\right)^{n_{1}{ }^{\prime}}\left(Z-b_{2}^{\prime}\right)^{n_{2}{ }^{\prime}} \cdots\left(Z-b_{\nu}^{\prime}\right)^{n_{\nu}{ }^{\prime}}}, \\
& \sum_{j=1}^{\nu} n_{j}^{\prime}=1,
\end{aligned}
$$

and whose boundary consists of mutually disjoint Jordan curves $C_{1}^{\prime}, C_{2}^{\prime}, \cdots, C_{\nu}^{\prime}$ which separate the $b_{j}^{\prime}$ respectively from $\Delta$. If there exists $a(1,1)$ conformal mapping of $D$ onto $\Delta$ so that the $a_{j}$ correspond respectively to the $a_{j}^{\prime}$, then this correspondence can be continued beyond $D$ and $\Delta$ as a linear transformation of the $z$-sphere onto the $Z$-sphere. We have $n_{j}=n_{j}^{\prime}$, and if arg $A^{\prime}$ is suitably chosen, $R(z) \equiv R_{1}(Z)$.

There is no essential difference in the proof of Theorem 4 from that of Theorem 2.

\section{BIBLIOGRAPHY}

1. James A. Jenkins, A general coefficient theorem, Trans. Amer. Math. Soc. vol. 77 (1954) pp. 262-280.

2. James A. Jenkins and D. C. Spencer, Hyperelliptic trajectories, Ann. of Math. vol. 53 (1951) pp. 4-35.

3. J. L. Walsh, On the conformal mapping of multiply connected regions, Trans. Amer. Math. Soc. vol. 82 (1956) pp. 128-146.

UNIVERSITY OF NOTRE DAME, Notre Dame, Ind. 